\title{
Endovascular Therapy versus Thrombolysis in Patients with Mild Strokes and Large Vessel Occlusions within the Anterior Circulation
}

\author{
Andreas Kastrup ${ }^{a, b} \quad$ Freimuth Brunner ${ }^{a}$ Helmut Hildebrandt ${ }^{a}$ \\ Christian Roth $^{c}$ Michael Winterhalter ${ }^{d}$ Carsten Giessing ${ }^{e}$ \\ Panagiotis Papanagiotou ${ }^{\mathrm{C}}$ \\ ${ }^{a}$ Department of Neurology, Klinikum Bremen-Mitte, Bremen, Germany; ${ }^{b}$ Department of \\ Neurology, University of Göttingen, Göttingen, Germany; ${ }^{C}$ Department of Neuroradiology, \\ Klinikum Bremen-Mitte, Bremen, Germany; ${ }^{d}$ Department of Anesthesiology, Klinikum \\ Bremen-Mitte, Bremen, Germany; ${ }^{e}$ Department of Psychology, Carl von Ossietzky \\ University Oldenburg, Oldenburg, Germany
}

\section{Keywords}

Stroke · Thrombolysis · Thrombectomy · Outcome - Endovascular treatment · Mild stroke

\begin{abstract}
Background: In patients with large vessel occlusions, endovascular treatment (ET) has been shown to be superior to intravenous thrombolysis (IVT) in recent trials. However, it is currently unclear if patients with mild strokes also benefit from ET. Methods: We compared the discharge rates of good outcome (modified Rankin scale [mRS] $\leq 2$ ), very good outcome (mRS $0-1)$, symptomatic intracranial hemorrhages $(\mathrm{SICH})$, and infarct sizes in patients with mild strokes (admission National Institutes of Health Stroke Scale $\leq 10$ ) and distal intracranial carotid artery, M1, and M2 occlusions during two time periods. Results: From 1/2008 to 10/2012 160 patients (mean age: $72 \pm 12$ years) were treated with IVT, and from 11/2012 to 11/2016 145 patients (mean age: $71 \pm 13$ years, ) received ET with or without IVT. The clinical results were comparable between both treatment groups (59\% after ET vs. $56 \%$ after IVT, $p=0.5$ for an mRS $0-2)$ and (38\% after ET vs. $32 \%$ after IVT, $p=0.3$ for an mRS $0-1)$. In the subgroup of patients with an $\mathrm{mRS} \leq 6$, the early outcome did not differ significantly between ET and IVT either. The rates of $\mathrm{SICH}$ as well as the infarct sizes were not significantly different after ET compared with IVT. Conclusion: Compared with IVT, the routine use of ET did not significantly
\end{abstract}

Andreas Kastrup and Freimuth Brunner contributed equally to this work.

Andreas Kastrup, MD

Department of Neurology, Klinikum Bremen-Mitte

St.-Jürgen-Strasse 1

DE-28177 Bremen (Germany)

E-Mail akastrup06@gmail.com 
improve the early clinical or radiological outcome in patients with mild strokes and anterior circulation large vessel occlusions. Further randomized trials are urgently needed to determine the role of ET in this cohort.

(C) 2018 S. Karger AG, Basel

\section{Introduction}

Based on the results of several recently published landmark trials, endovascular treatment (ET) (with the use of third-generation retrievable stents in the majority of patients) has become the treatment of choice in patients with proximal intracranial occlusions of the anterior circulation [1]. However, in several of these trials, the patients were highly selected and those with minor strokes were either excluded or underrepresented [2-4]. In a meta-analysis of individual patient data from 5 trials, ET was not significantly better than intravenous thrombolysis (IVT) in a total of 177 patients with an admission National Institutes of Health Stroke Scale (NIHSS) $\leq 10$ (OR: 1.67; 95\% CI: 0.80-3.50) [5]. In a recently published small single-center study with 54 patients with a proximal intracranial occlusion within the anterior circulation and an admission NIHSS $\leq 5$, the rates of an excellent clinical outcome (modified Rankin Scale [mRS] 0-1 after 3 months) tended to be higher after ET than after IVT [6]. High rates of an excellent clinical outcome after ET in this patient population have also been reported by Dargazanli et al. [7], however without comparing these results with IVT. Therefore, the question arises if ET is superior to IVT in patients with mild or minor strokes. Using our prospectively obtained database of a high-volume stroke center, we compared the early clinical and radiological outcome in patients with a mild stroke (admission NIHSS $\leq 10)$ and anterior circulation large vessel occlusions during two time periods. During the first time period, all patients had been treated with systemic thrombolysis, whereas an endovascular therapy with stent retrievers (with or without systemic thrombolysis) was routinely used during the second time period.

\section{Methods}

\section{Study Population}

We identified patients with distal intracranial carotid artery and/or M1 and/or M2 segments of the middle cerebral artery and an admission NIHSS $\leq 10$, who had been treated from January 2008 through November 2016. Patients with distal occlusions beyond the M2 segment were excluded. In addition, patients with posterior circulation strokes and with bilateral strokes were excluded from the current analysis. Before November 2012, all patients were treated with systemic thrombolysis within 4.5 h. After November 2012, a systematic approach (using no upper age limit or specific imaging exclusion criteria) comprising ET with and without systemic thrombolysis was implemented in patients who presented within $6 \mathrm{~h}$ (within $4.5 \mathrm{~h}$ in patients additionally treated with rt-PA) of symptom onset. These patients were treated with retrievable stents: Solitaire FR (Covidien); pREset (Phenox); Catch+ (Balt).

Demographic data (age, gender) and stroke risk factors (arterial hypertension, diabetes, hyperlipidemia, atrial fibrillation) were collected. The time to thrombolysis (or to thrombectomy in patients without prior systemic thrombolysis) from stroke onset was also noted.

The protocol of our stroke registry had been approved by our local ethics committee. Because of the retrospective character of this study, the lack of treatment influence, and the clinical data having been collected as part of a national quality control program, the study was exempt from informed consent. Using our stroke database, the clinical outcomes of patients treated before December 2014, as well as a subgroup analysis have been published previously $[8,9]$.

Imaging Techniques

Nonenhanced CT and CT angiographic acquisitions before treatment were performed on a 4-row Multisection CT scanner (Siemens Volume Zoom, Siemens Medical Solutions, Forchheim, Germany). 
Nonenhanced CT was performed with the patient in a head holder in the transverse plane. Using the following parameters, incremental CT acquisitions of the brain were obtained: $120 \mathrm{kVp}, 250 \mathrm{~mA}, 2$-s scan time, and 5-mm section thickness.

To allow visualization of the vascular tree from the distal common carotid artery to the intracranial vessels, the CT angiography covered the region from the fifth vertebral body up to the vertex. The following parameters were used: $120 \mathrm{kVp}, 200 \mathrm{mAs}, 4 \times 1-\mathrm{mm}$ collimation, $5.5 \mathrm{~mm} /$ rotation table feed, and $0.5 \mathrm{~s}$ rotation time. A total of $100 \mathrm{~mL}$ of contrast material was injected intravenously at a flow rate of $4 \mathrm{~mL} / \mathrm{s}$ using a power injector. For follow-up studies, repeated CT or MR scans were obtained after 1-3 days after treatment or immediately in case of clinical worsening.

Image Analysis

CT and MR image analyses were performed jointly by a board-certified neuroradiologist (P.P., 14 years of neuroimaging review experience) and a stroke neurologist (A.K. with 16 years of neuroimaging review experience) on a high-resolution monitor.

The Alberta Stroke Program Early CT score (ASPECTS) is a 10-point quantitative topographic CT scan score with 1 point subtracted for evidence of ischemic change in each defined region. In this study, the CTAsource images were used to determine the CTA-SI-ASPECTS [10]. The follow-up CT or MR scans were used to determine the final ASPECTS as a marker of infarct extent, as well as the incidence of symptomatic intracranial hemorrhages (SICH) using the ECASS III definition.

\section{End Points}

Clinical outcome measures were a favorable early outcome (mRS of $0-2$ at the time of discharge), an excellent early outcome (mRS of $0-1$ at the time of discharge), and a poor outcome (mRS of 5-6 at the time of discharge). Imaging outcome measures included the extent of the infarcts and SICH rates.

\section{Statistical Analysis}

Normally distributed data are presented as mean \pm SD and nonnormally distributed data as median and interquartile range. Categorical variables are expressed as percentages. For comparisons of categorical data, two-tailed $\chi^{2}$ statistics with Yates correction was used. Analyses of continuous variables were performed with an unpaired Student's $t$ test or in case of abnormally distributed data with a Mann-Whitney U test. In a first step, multivariable logistic regression was performed using standard techniques to model good outcome $(\mathrm{mRS} \leq 2)$ and excellent outcome ( $\mathrm{mRS} \leq 1)$. These statistical analyses were performed with SPSS (v22, SPSS Inc). In a second step, we performed a nonparametric propensity score matching analysis [11]. Within a first step, potentially confounding variables ("age," "sex," "admission NIHSS," "occlusion site," and "the admission SI-ASPECTS") were included within a logistic regression model to estimate a propensity score. These propensity scores were used as distance measure to select for each individual within the ET group the nearest matching subject within the IVT group inside a range of 0.25 standard deviation. Based on these matched treatment groups, treatment-related effects on the number of patients with good outcome (mRS $\leq 2)$ and excellent outcome $(\mathrm{mRS} \leq 1)$ were analyzed with two-tailed $\chi^{2}$ tests with Yates correction. Propensity score matching was performed with the R-statistics package "MatchIt" (https://www.r-project.org/).

\section{Results}

\section{Demographic Data}

From January 2008 to November 2016, a total of 1,038 patients with distal intracranial carotid artery, M1 and M2 occlusions had been treated with systemic thrombolysis (IVT) or endovascular therapy (ET) at our institution. The study group comprised 305 patients (29\%), who had had an admission NIHSS $\leq 10$. There were 132 males (43\%) and 173 females $(57 \%)$ with a mean age of $71 \pm 13$ years. Among these, 160 patients had been treated with systemic thrombolysis (IVT group), and a total of 145 patients had received an endovascular therapy (ET group), respectively. In the ET group, 48 patients (33\%) had had contraindications for a systemic thrombolysis and thus were solely treated with ET. Table 1 summarizes the baseline characteristics of both treatment groups. There were no significant differences in risk factors 
Table 1. Patient characteristics according to treatment

\begin{tabular}{|c|c|c|c|}
\hline & $\begin{array}{l}\text { Endovascular } \\
\text { therapy } \\
(n=145)\end{array}$ & $\begin{array}{l}\text { Thrombolysis } \\
\text { alone } \\
(n=160)\end{array}$ & $p$ value \\
\hline Age, years & $71 \pm 13$ & $72 \pm 12$ & 0.4 \\
\hline Male & $65(45)$ & $67(42)$ & 0.6 \\
\hline \multicolumn{4}{|l|}{ Risk factors } \\
\hline Hypertension & $122(84)$ & $129(81)$ & 0.4 \\
\hline Hyperlipidemia & $37(25)$ & 37 (23) & 0.6 \\
\hline Diabetes mellitus & $32(22)$ & $35(22)$ & 1.0 \\
\hline Atrial fibrillation & $57(39)$ & $74(46)$ & 0.2 \\
\hline NIHSS & $8(5-9)$ & $7(5-9)$ & 0.4 \\
\hline \multicolumn{4}{|l|}{ Workflow times, min } \\
\hline Stroke onset to imaging & $125(105-152)$ & $130(106-150)$ & 0.9 \\
\hline Stroke onset to initiation of systemic thrombolysis & $130(115-170)$ & $150(120-170)$ & 0.2 \\
\hline CT to groin puncture & $60(50-70)$ & NA & \\
\hline \multicolumn{4}{|l|}{ Location of stroke } \\
\hline Left hemisphere & $72(50)$ & $72(45)$ & 0.4 \\
\hline Right hemisphere & $73(50)$ & $88(55)$ & \\
\hline \multicolumn{4}{|l|}{ Location of occlusion ${ }^{a}$} \\
\hline Intracranial ICA & $32(22)$ & $19(12)$ & $<0.05$ \\
\hline M1 MCA & $62(43)$ & $76(47)$ & 0.4 \\
\hline M2 MCA & $51(35)$ & $65(41)$ & 0.3 \\
\hline Baseline SI-ASPECTS & $9(8-10)$ & $10(9-10)$ & $<0.01$ \\
\hline
\end{tabular}

Data are presented as mean $\pm \mathrm{SD}$, median (IQR), or $n(\%)$. NIHSS, National Institutes of Health Stroke Scale; SI-ASPECTS, Source image Alberta Stroke Program Early CT score; ICA, internal carotid artery; MCA, middle cerebral artery. ${ }^{\text {a }}$ Most proximal occlusion location.

between both treatment groups. Overall, the most proximal occlusion was located in the distal intracranial carotid artery in $51(17 \%)$ patients, in the M1 segment of the middle cerebral artery in 138 (45\%) patients, and in the M2 segment in 116 (38\%) patients, respectively. Compared with the IVT group, more patients had a distal intracranial carotid artery occlusion in the ET group. In addition, early signs of ischemia were significantly more pronounced in the ET group than in the IVT group (admission SI-ASPECTS 9, IQR: 8-10 in the ET group vs. admission SI-ASPECTS 10, IQR: 9-10 in the IVT group; $p<0.01$ ), which could be attributable to a notable number of patients 17/145 (12\%) with an unknown stroke onset time in the ET group.

\section{Clinical Outcome}

Overall, 175/305 patients (57\%) had a favorable early clinical outcome (mRS 0-2), $105 / 305$ patients (34\%) had an excellent early clinical outcome (mRS 0-1), and 31/305 patients (10\%) had a poor clinical outcome (mRS 5-6), respectively. These outcome variables were comparable between both treatment groups (Table 2). The clinical results were also comparable between both treatment groups after excluding the 48 patients with a contraindication for a systemic thrombolysis in the ET group (60\% after ET vs. $56 \%$ after IVT, $p=0.5$ for an mRS $0-2 ; 40 \%$ after ET vs. $32 \%$ after IVT, $p=0.2$ for an mRS $0-1$ ).

We performed an additional subgroup analysis to assess the effect of treatment on clinical outcome in patients with a very mild stroke, defined as an admission mRS $\leq 6$. In the IVT group, $67 / 160(42 \%)$ patients had had a very mild stroke (mRS $\leq 6)$ and in the ET group $50 / 145$ (34\%) patients, respectively. In good agreement with the results for the entire study 
Table 2. Clinical and radiological outcomes according to treatment

\begin{tabular}{llll}
\hline & $\begin{array}{l}\text { Endovascular } \\
\text { therapy }(n=145)\end{array}$ & $\begin{array}{l}\text { Thrombolysis } \\
\text { alone }(n=160)\end{array}$ & $p$ value \\
\hline $\begin{array}{l}\text { Clinical outcome } \\
\text { mRS 0-2 }\end{array}$ & $86(59)$ & $89(56)$ & 0.5 \\
mRS 0-1 & $55(38)$ & $52(32)$ & 0.3 \\
mRS 5-6 & $19(13)$ & $22(15)$ & 0.9 \\
$\begin{array}{l}\text { Radiological outcome } \\
\text { SICH }\end{array}$ & $3(2)$ & $6(4)$ & 0.4 \\
Follow-up ASPECTS & $8(7-9)$ & $8(6-9)$ & 0.6 \\
\hline
\end{tabular}

Data are presented as $n(\%)$ or median (IQR). mRS, modified Rankin Scale; SICH, symptomatic intracerebral hemorrhage; ASPECTS, Alberta Stroke Program Early CT score.

Table 3. Effects of propensity score matching on the balance of confounds

\begin{tabular}{lcccccc}
\hline & \multicolumn{2}{l}{ All data } & & \multicolumn{2}{l}{ Matched data } & \multirow{2}{*}{$\begin{array}{l}\text { Percent balance } \\
\text { improvement }\end{array}$} \\
\cline { 2 - 3 } & ET, means & IVT, means & & ET, means & IVT, means & \\
\hline Distance & 0.50 & 0.47 & & 0.48 & 0.48 & 75.49 \\
Age & 70.22 & 71.60 & & 70.86 & 71.19 & 75.38 \\
Female & 0.55 & 0.58 & & 0.55 & 0.57 & 38.25 \\
NIHSS & 7.27 & 6.96 & & 7.17 & 7.11 & 80.94 \\
M2 occlusion & 0.42 & 0.47 & & 0.44 & 0.47 & 31.16 \\
SI-ASPECTS & 8.69 & 9.18 & & 8.97 & 9.07 & 79.02 \\
\hline
\end{tabular}

The balance of confounding variables between both treatment groups (endovascular therapy - ET vs. intravenous thrombolysis - IVT) improved following propensity score matching. ET, IVT, percent balance improvement: the percent reduction in the difference in means achieved in each of the balance measures. NIHSS, National Institutes of Health Stroke Scale; SI-ASPECTS, Source image Alberta Stroke Program Early CT score.

population, the early outcome did not differ significantly between ET and IVT (64 vs. $72 \%$, $p=0.4$ for an mRS $0-2 ; 42$ vs. $48 \%, p=0.5$ for an mRS $0-1$ ).

In the multivariate regression analysis, only admission NIHSS (OR: 0.84, 95\% CI: $0.76-$ 0.93; $p<0.001$ for an mRS $0-2$ and OR: $0.83,95 \%$ CI: 0.76-0.92; $p<0.001$ for an mRS $0-1$ ) and age (OR: 0.97, 95\% CI: 0.95-0.99; $p<0.001$ for an mRS 0-2 and OR: 0.98; 95\% CI: 0.96$0.99 ; p<0.05$ for an mRS $0-1$ ) were significantly associated with a good or excellent early clinical outcome.

\section{Propensity Score Matching}

The propensity score matching procedure improved the balance of potentially confounding factors (age, sex, admission NIHSS, site of occlusion, and admission SI-ASPECTS) between both treatment groups (Table 3). Following propensity score matching, the number of patients with good outcome (mRS 0-2) or excellent outcome (mRS 0-1) did not significantly vary between both treatment groups (ET vs. IVT; good outcome vs. no good outcome: $\chi^{2}=0.63, \mathrm{df}=1, p=0.43$; excellent outcome vs. no excellent outcome: $\chi^{2}=0.89, \mathrm{df}=1, p=$ 0.35 ). 


\section{Radiological Outcome}

Follow-up imaging was not available in 37 patients. In good agreement with the clinical results, the infarct sizes were comparable between both treatment groups (follow-up ASPECTS 8, IQR: 6-9 in the IVT group vs. follow-up ASPECTS 8, IQR: 7-9 in the ET group; $p=$ $0.6)$.

For the entire cohort, the overall incidence of symptomatic hemorrhages (using the ECASS III definition) was $9 / 305$ (3\%). The SICH rates were comparable between both treatment groups $(6 / 160$ in the IVT group vs. $3 / 145$ in the ET group, $p=0.4)$.

\section{Discussion}

Based on a large prospectively recorded database of a high-volume academic stroke center and two time periods of treatment, the goal of this study was to compare the clinical and radiological outcome in patients with mild strokes and large-vessel intracranial occlusions within the anterior circulation after systemic thrombolysis or endovascular therapy with modern stent retrievers. Despite the fact that approximately $50 \%$ of these patients had had an acute occlusion of the internal carotid artery and/or the M1 portion of the middle cerebral artery, the routine use of endovascular therapy did not significantly improve the early clinical or radiological outcome in this study.

The Central Hospital of Bremen is an acute care 900-bed teaching hospital with the only stroke unit in the city of Bremen serving a population of approximately 750,000 people. At our institution, all patients with suspicion of acute stroke and no history of renal insufficiency or contrastallergy routinely undergo acute CT angiography at the time of admission. Therefore, our database offers the unique opportunity to analyze the magnitude of the problem dealing with the optimal treatment of patients with mild strokes and acute proximal vessel occlusions. The fact that nearly one-third of the patients with a large vessel occlusion within the anterior circulation had had an admission NIHSS $\leq 10$ and $11 \%$ of the patients admitted to our hospital even had had an NIHSS $<6$ during the study period supports the notion that the question if ET is superior to IVT in these patients is clinically highly relevant.

Irrespective of the treatment modality, $57 \%$ of the patients had a good early clinical outcome, and $35 \%$ of the patients had an excellent early clinical outcome. However, even after excluding patients with contraindications for a thrombolysis, the clinical outcome was not significantly different between ET and IVT in patients with an admission NIHSS $\leq 10$, as well as in patients with an admission NIHSS $<6$. In good agreement with the clinical data, the extent of ischemia was also comparable between both treatment modalities. While a subgroup analysis of individual patient data of 5 randomized trials principally showed no heterogeneity in the positive treatment effect of ET compared with IVT across the range of baseline NIHSS values, the point estimate of ET on outcome in patients with an NIHSS $\leq 10$ showed a wide confidence interval (OR: 1.67; 95\% CI: 0.80-4.50) [5]. In addition, 4 out of 5 trials used the baseline NIHSS scores as eligibility criteria $(>2,>5,8-29$, and $>5)$ so that only very few patients had a mild stroke in these studies [5]. Despite the fact that patients with major vessel occlusion and mild clinical deficits at presentation have been shown to have a substantial risk of subsequent deterioration [12], our data do not support the concept that ET improves outcome in these patients.

Strengths of our study include the large sample size, as well as the homogeneity of the sample from a single institution and having been evaluated and treated by the same stroke team. However, certain limitations of the present report need to be acknowledged. First, although we used a prospectively collected cohort, the impact of ET or IVT on early clinical and radiological outcome was determined in a nonrandomized and retrospective fashion. 
Hence, the study holds all the drawbacks of observational design. Second, the discharge mRS served as markers of early clinical outcome, while the time point generally recognized as most appropriate to assess functional outcome after ischemic stroke is 3 months. On the other hand, a recent reanalysis of the NINDS tPA stroke trial has indicated that the day $7 \mathrm{mRS}$ can serve as a good proxy for the 90-day mRS [13]. Third, the status of recanalization was not determined routinely in the IVT group, so that this important variable was not considered in our analyses. Finally, many patients had had an M2 occlusion; thus, our results might not be generalizable to patient populations with higher rates of more proximal large vessel occlusions.

In conclusion, the routine use of ET did not significantly improve the early clinical or radiological outcome in patients with mild strokes and anterior circulation large vessel occlusions in this study compared with IVT despite the fact that every second patient had an acute occlusion of the internal carotid artery and/or the M1 portion of the middle cerebral artery. Nearly one-third of the patients treated in our high-volume stroke center had an admission NIHSS $\leq 10$ and approximately $10 \%$ of the patients an admission NIHSS $\leq 6$, indicating that it is clinically highly relevant to further determine the role of ET in this cohort. Although it is beyond doubt that a randomized trial is needed, the difference in outcome between these two treatment modalities in these patients, if any at all, is small so that only a large trial will be able to answer this important question.

\section{Disclosure Statement}

The authors declare that they have no conflicts of interest.

\section{Funding Sources}

None.

\section{References}

1 Powers WJ, Derdeyn CP, Biller J, Coffey CS, Hoh BL, Jauch EC, Johnston KC, Johnston SC, Khalessi AA, Kidwell CS, et al: 2015 American Heart Association/American Stroke Association Focused Update of the 2013 Guidelines for the Early Management of Patients with Acute Ischemic Stroke Regarding Endovascular Treatment: A Guideline for Healthcare Professionals from the American Heart Association/American Stroke Association. Stroke 2015;46:3020-3035.

2 Goyal M, Demchuk AM, Menon BK, Eesa M, Rempel JL, Thornton J, Roy D, Jovin TG, Willinsky RA, Sapkota BL, et al: Randomized assessment of rapid endovascular treatment of ischemic stroke. N Engl J Med 2015;372: 1019-1030.

3 Jovin TG, Chamorro A, Cobo E, de Miquel MA, Molina CA, Rovira A, San Roman L, Serena J, Abilleira S, Ribo M, et al: Thrombectomy within 8 hours after symptom onset in ischemic stroke. N Engl J Med 2015;372:22962306.

4 Saver JL, Goyal M, Bonafe A, Diener HC, Levy EI, Pereira VM, Albers GW, Cognard C, Cohen DJ, Hacke W, et al: Stent-retriever thrombectomy after intravenous t-PA vs. t-PA alone in stroke. N Engl J Med 2015;372:22852295.

5 Goyal M, Menon BK, van Zwam WH, Dippel DWJ, Mitchell PJ, Demchuk AM, Dávalos A, Majoie CBLM, van der Lugt A, de Miquel MA, et al: Endovascular thrombectomy after large-vessel ischaemic stroke: a meta-analysis of individual patient data from five randomised trials. Lancet 2016;387:1723-1731.

6 Messer MP, Schonenberger S, Mohlenbruch MA, Pfaff J, Herweh C, Ringleb PA, Nagel S: Minor stroke syndromes in large-vessel occlusions: mechanical thrombectomy or thrombolysis only? AJNR Am J Neuroradiol 2017;38: 1177-1179.

7 Dargazanli C, Consoli A, Gory B, Blanc R, Labreuche J, Preda C, Bourdain F, Decroix JP, Redjem H, Ciccio G, et al: Is reperfusion useful in ischaemic stroke patients presenting with a low National Institutes of Health Stroke Scale and a proximal large vessel occlusion of the anterior circulation? Cerebrovasc Dis 2017;43:305-312. 
8 Wasser K, Papanagiotou P, Brunner F, Hildebrandt H, Winterhalter M, Roth C, Kastrup A: Impact of ASPECTS on computed tomography angiography source images on outcome after thrombolysis or endovascular therapy in large vessel occlusions. Eur J Neurol 2016;23:1599-1605.

9 Kastrup A, Brunner F, Wasser K, Hildebrandt H, Roth C, Winterhalter M, Papanagiotou P: Endovascular therapy versus thrombolysis in patients with anterior circulation stroke in everyday clinical practice. Int J Stroke 2016;11:544-548.

10 Coutts SB, Lev MH, Eliasziw M, Roccatagliata L, Hill MD, Schwamm LH, Pexman JH, Koroshetz WJ, Hudon ME, Buchan AM, et al: ASPECTS on CTA source images versus unenhanced CT: added value in predicting final infarct extent and clinical outcome. Stroke 2004;35:2472-2476.

11 Ho DE, Imai K, King G, Stuart EA: Matching as nonparametric preprocessing for reducing model dependence in parametric causal inference. Political Analysis 2007;15:199-236.

12 Coutts SB, Modi J, Patel SK, Aram H, Demchuk AM, Goyal M, Hill MD: What causes disability after transient ischemic attack and minor stroke? Results from the CT and MRI in the Triage of TIA and minor Cerebrovascular Events to Identify High Risk Patients (CATCH) Study. Stroke 2012;43:3018-3022.

13 Ovbiagele B, Saver JL: Day-90 acute ischemic stroke outcomes can be derived from early functional activity level. Cerebrovasc Dis 2010;29:50-56. 